IJBPAS, November, Special Issue, 2021, 10(11): 725-736

ISSN: 2277-4998

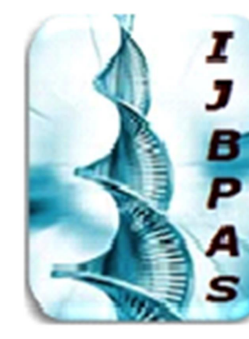

International Journal of Biology, Pharmacy and Allied Sciences (IJBPAS)

'A Bridge Betueen caboratory and QRendo'

Www.ijbas.com

\title{
MACHINE LEARNING APPROACH FOR PROTECTING THE INFORMATION TRAFFIC WITH FIREWALL FOR SMART IOT DEVICES AND NETWORK
}

\section{TEJA SIRAPU ${ }^{*}$, NANDHINI. ${ }^{2}$, SURESH KUMAR K $^{3}$, GUNA SEKHAR SAJJA ${ }^{4}$, RAVI KUMAR SAIDALA ${ }^{5}$ AND LOKESH M R ${ }^{6}$}

1: Assistant Professor in Electronics and communication engineering at Shri Vishnu Engineering College for Women (Autonomous), Bhimavaram, West Godavari Andhra Pradesh, India

2: Assistant Professor in MCA at SNS College of Technology, Sathy Main Road, Vazhlampalayam Pirivu, Coimbatore, Tamilnadu, India

3: Associate Professor in MBA at Panimalar Engineering College, Varadarajapuram, Nasarathpettai, Poonamallee, Chennai India

4: Research scholar at Information Technology Department, University of the Cumberlands, Kentucky, USA

5: Associate Professor, Department of Information Technology, Vishnu Institute of Technology, Bhimavaram, Andhra Pradesh 534202, India

6: Professor in Computer Science and Engineering at Maharaja Institute of Technology

Mysore, Mandya, karnataka, India

*Corresponding Author: Teja Sirapu; E Mail: siraputeja@gmail.com

Received 20 ${ }^{\text {th }}$ July 2021; Revised 22 ${ }^{\text {nd }}$ Aug. 2021; Accepted 30 ${ }^{\text {th }}$ Sept. 2021; Available online $1^{\text {st }}$ Nov. 2021 https://doi.org/10.31032/IJBPAS/2021/10.11.1062

\begin{abstract}
Internet enables billions of devices, posing a range of issues as possibilities. An exponential growth in the number of internet - connected devices of Things (IoT) would be almost unfathomable. These nodes transmit each other, making human existence easier. This interconnection of these gadgets has paved way for intelligent systems that were a rapidly increasing field of study. Confidentiality and anonymity were regarded to be among the most
\end{abstract}


important challenges that academics to address among these prospects. Aggressors would be unable to disrupt integrity of IoT network within the modern city for protected data stream if proper safety controls are adopted. A suggested research provided classification technique for safeguarding internet traffic predicated on a gateway for digital sensors on IoT networks, bearing in mind the security aspects of data centres for digital sensors or IoT. A combination proposed approach surpasses decision 1 rules or arbitrary forest, yielding a detection performance of 95.5 percent for hybrid version, 68.5 percent using majority voting, or 78.3 percent reliability for arbitrary forest, as per approach's analytical outcomes. Other acceptance criteria such as $f$ value, confidence interval, retention, or controllability should be used to check the feasibility of proposed methodology. A suggested combination designer's high precision score or other quality measures demonstrate its suitability for secure information traffic applications in digital sensors. This could be used for protective measures in a range of smart metropolis application fields.

\section{Keywords: Internet of Things (IoT); Artificial Intelligence Techniques (MLA);}

\section{Intelligent Buildings; Communication Traffic}

\section{INTRODUCTION}

A interconnection of these gadgets has opened up new avenues for intelligent systems, which is among the fastestgrowing research fields [1]. Confidentiality and anonymity were regarded to be one of most important challenges for academics to address among prospects. Urban civilizations have a clear need for smart communication [2]. Internet of Things (IoT) plays an important role as in amazing skills of these gadgets [3]. Innovation has mostly emphasized on excellent human very well ecosystem preservation [4].

IoT technology has limitless possibilities for creating environmentally friendly yet intelligent gadgets. In most cases, data is collected via physical things or dispersed during processing through digital communication [5]. For data acquisition, high-performance computing methods are employed [6]. These techniques can help a municipal government provide critical information for optimum adsorbent operation [7]. Internet of Things (IoT) developed and maintained that clever products are provided using efficient asset allocation. A doorway for cognitive processing or computerized administration of pervasive computing was established focused on IoT systems [8-10]. As a consequence, encryption could be regarded a critical aspect of IoT network for smart urban [11] in order to preserve overall 
seamless coordination and engagement of gadgets as in IoT network.

In smart urban, alternative approaches of protection interaction were used. [12] Established a framework with assist of an Arduino-programmed detector or uploaded visual information to a cloud services system to obtain access to electronic connected devices. [13] proposed a model for evaluating a broadcaster's practicality or functionality as in event of a connection breakdown or switching to a regular scenario. A performance of the proposed approach was evaluated using a variety of criteria [14]. These investigations revealed that overall was capable of recognizing or mitigating assaults, indicating that it could be used to secure a structure to ensure device security.

Urban areas urgently require actual precautions to prevent assailants in disrupting overall integrity of IoT network within modern city, provided data of gadgets across internet traffic was secure. A suggested analysis made innovations, bearing in mind overall security aspects of internet traffic for smart urban or IoT:

(i) Demonstrate classification techniques of safeguarding internet data in digital sensors or IoT networks using a gateway. (ii) To employ the "Router" database for submitted article's assessment.

(iii) To demonstrate a feasibility of proposed method via investigations.

(iv) Other quantitative measurements such as $f$ value, margin of error, retention, or accuracy are used to assess overall stability of model.

(v) A detection performance or other quality measures demonstrate the proposed combination designer's suitability to protected data flow in digital sensors.

.Investigators were attempting to develop diverse methods, tactics, or procedures of solving critical safety issues in smart urban. A designer's method comprises of creating a model to proper approach, deriving sequences from concept, applying divergence guidelines for assessing testing procedures, running the trials, as well as finally collecting outcomes or assessing them to find or correct issues. An accepted framework was predicated on the prolonged delayed automata to inputs and outputs design. Challenges, problems, or processes related to IoT safety. The report also detailed the potential security risks to IoT devices in a smart urban scenario. 
Material on the structure, framework, innovation, or specific applications of IoT smart urban [15]. A greatest IoT has a number of issues in level of protection, end-to-end connectivity, or power efficiency. The flaws in best-effort IoT security were exposed, or a safe probabilistic commercial hepatic IoT network was demonstrated.

The traffic monitoring assault for connected devices, in which adversaries disrupt traffic from to the home automation entrance or use digital evidence to analyse occupants' behaviour. Due to capability of adversaries, conventional cryptographic methods are often not practicable. For reaching this goal, study provided a paradigm for privacy-preserving obscuring. A proposed method framework was tested using several simulations. The findings demonstrated the platform's performance in comparison to other techniques. The Privacy or security to smart city of healthcare application. A study begins with a thorough examination of various IoT applications including their digital dangers, followed by a thorough examination of potential techniques for dealing with cyberattacks. Smart cities can currently be used in a variety of ways. For data propagation, these application cases include cooperating transport networks, automated driving, or clever roadways. An assessment of smart urban possibilities based on IoT connectivity for intelligent transport systems. A combination of cloud services and IoT for massive data was presented in this report [16]. A study aims to improve information security by demonstrating the system security design. IoT devices produce bandwidth based on the mentioned characteristics and differences from conventional channels. A study was presented to look at the feasibility of using these attributes for device classification. In situations of complexity or flexibility, this categorization was preferable. An aggregate of 41 IoT devices were used. A methodology was introduced as a feasible possibility for running a digital cellular $6 \mathrm{G}$ network in smart cities to monitor huge data.

Study aims to improve information security by demonstrating the system security design. IoT devices generate bandwidth based on distinct characteristics or differences from conventional channels. A study was presented to look just at feasibility of using attributes for device classification. In situations of complexity or flexibility, this categorization was preferable. An aggregate of 41 IoT devices were used. A methodology was introduced as a feasible possibility for running a digital cellular 6G network in a data center to monitor huge data. True positive rate, 
sensors capture speed, false-positive rate, end-to-end latency, and bandwidth were amongst these metrics. Findings showed that the suggested methodology was supported for adoption in an industrial Internet of things context.

A low-cost code distribution technique that disseminates updated codes using mobility automobiles across the metropolis using an adaptable speech pattern. A reportage pessimistic distribution technique was employed for code stations, and an approach of optimised code composition was applied to maximise code distribution penetration across the metropolis while reducing time and expense. Numerous tests were conducted to verify the theoretical inquiry, as well as findings proved that recommended approach is beneficial. Appropriate abnormality detection and classification for network identification based on IoT might be considered a crucial topic for researchers to address. An objective fuzzy soft method to feature extraction was established for picking relevant attributes, and a Perfect features extraction measure was developed. Additionally, the study established a new feature selection algorithm, Accurate, founded on Accurate, for identifying the most appropriate or productive characteristics for different classifiers using ACC score.

\section{Study on Securing Data Traffic of Smart Devices and IoT}

Smart urban inferences include data protection concerns. A prospective uses and repercussions on connected phones were explored in research works in this field. In recent months, a limited handful of concerns have received more attention than extortion operations. Malware and extortion that hide assets or deny entry to them until the author wants to pay. Among many associated parties extortion money were crypto locker, reverting, want torchy, and crypto wall.

There are many client classifications for which damages were hard to analyze, including conventional extortion victim, such as the loss of personal documents or critical labour related to financial data to family photos. A report provides a full categorization of significant concerns especially in the field and main technologies, as well as a detailed assessment that emphasizes on IoT architecture security. Acceptable standards of IoT infrastructure were examined, as well as distributed applications or venues for its creation. A unique method has been described in academic research, in which the IoT client can share part of verification functionality with IoT server.

For resolving numerous challenges in smart urban, many ways were used. A 
purpose of this study was to find existing empirical studies in the field. Several wellknown libraries were combed for the research's connected details. Various sorts of materials with published studies in scientific databases were visually represented in Figure 1. More articles in the discipline of Computing Science were produced, as seen in graph.

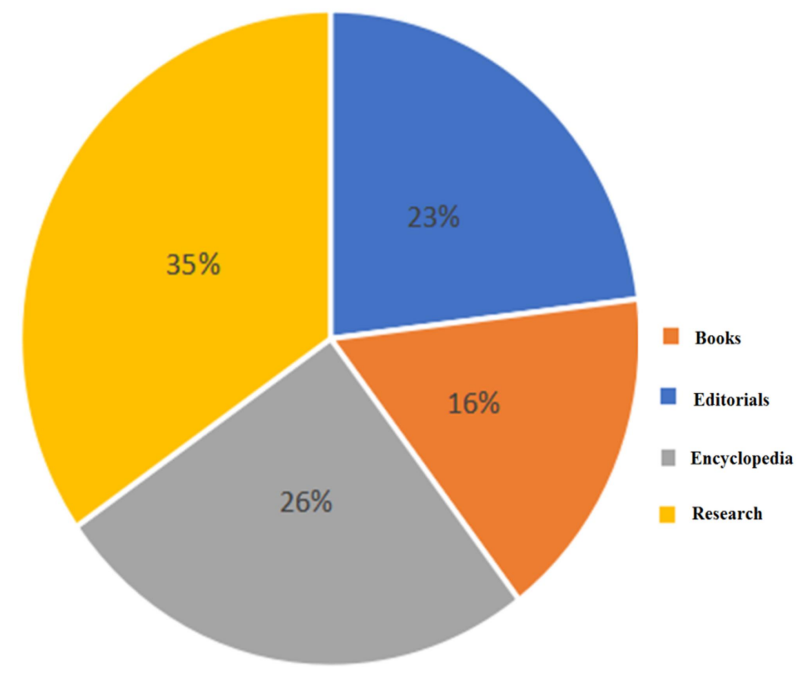

Figure 1: Types of Articles

RESULTS AND DISCUSSION

Springer archive was combed for information on current work in the domain.

Figure 2 shows various specialties represented as in location, as well as overall number of articles.

Figure 2 depicts the different categories of articles as well as overall number of articles as in specific region.

A documents for analytical procedure were found as in ACM collection. The publishing kinds or publications as in specified repository were depicted in Figure 3.

Figure 5 shows the overall number of articles presented at the meeting as in specific region.
Results were used to conduct a more in-depth examination of resources as in specified course of research. Figure 6 depicts a sample consisted used to display the library's publications.

IEEE library was examined, or findings were acquired for evaluation. Figure 7 shows different categories of publications or number of articles as in IEEE search.

This library was further investigated in order to acquire additional search terms. Figure 8 depicts various locations of conference sessions.

Findings of simulation studies were predicated on the Kaggle "Network Curtain" database. Python tool was used to 
carry out experiments. A usage of a combination learning algorithm centred on convolution networks (CNN) or support vector machines was adopted in this research (SVM). For segmentation, $\mathrm{CNN}$ is employed, while the SVM would be used for identification and forecast. Figure 9 depicts the performance analysis among various algorithms.
After evaluating the intended hybrid version for a variety of production measures such as validation accuracy, selectivity, F score, sensitivity, retention, or accuracy, it was determined that the composite system surpasses other classifying methods extremely well, as shown in Figure 9.

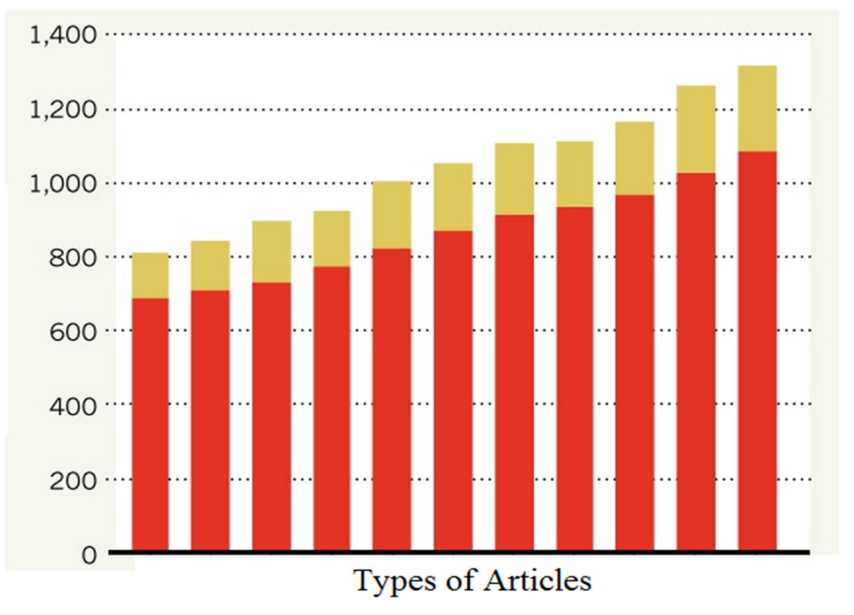

Figure 2: Publication of Articles

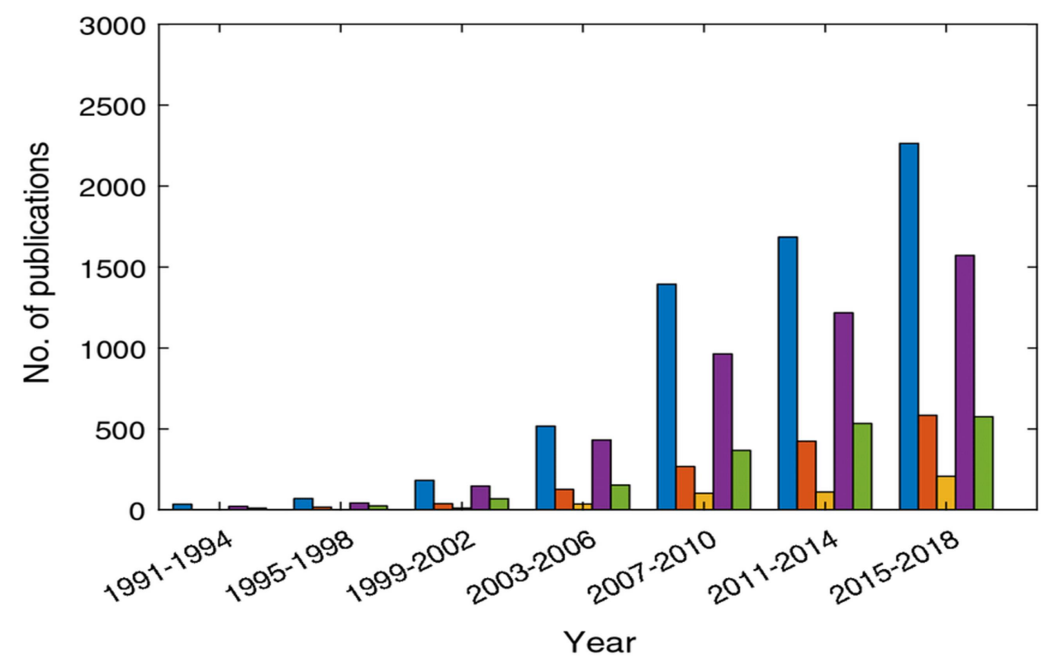

Figure 3: Publication analysis 


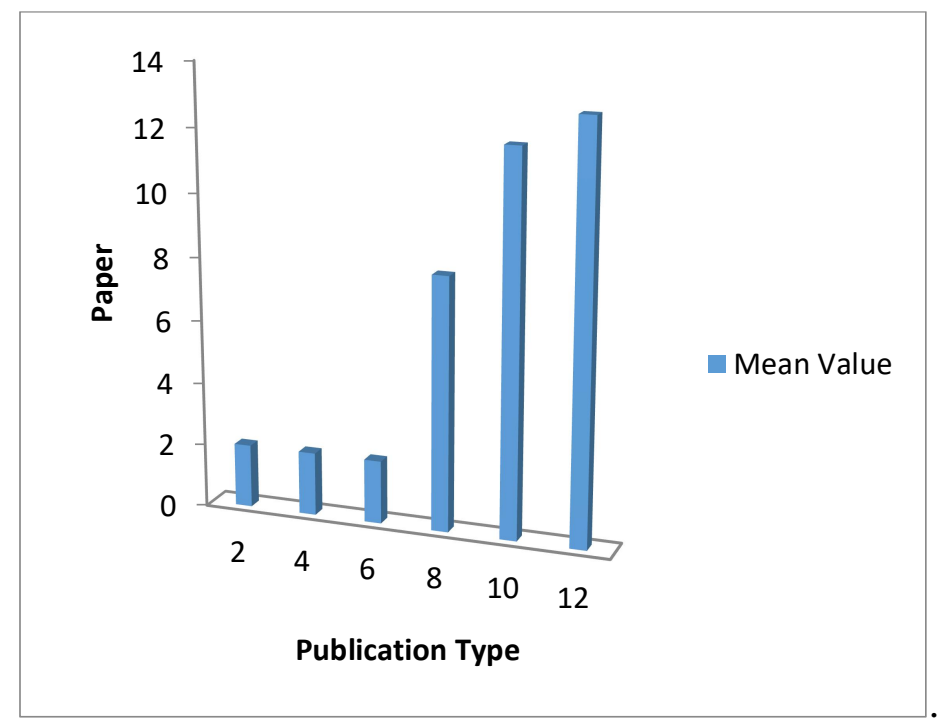

Figure 4: Types of Publications Vs Paper

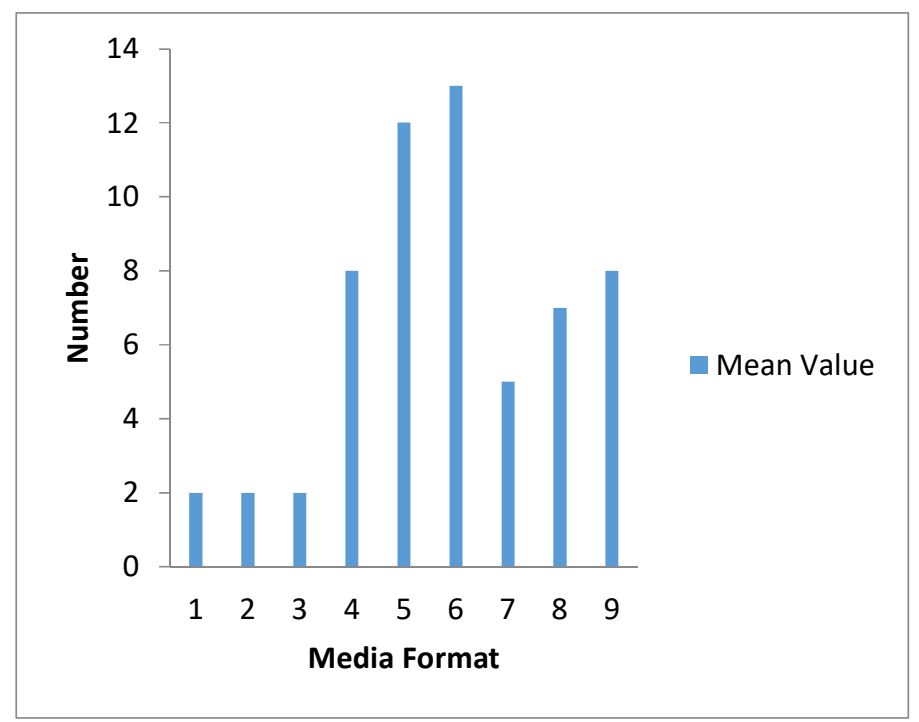

Figure 5: Mean Value of Types of Media Format Vs Number

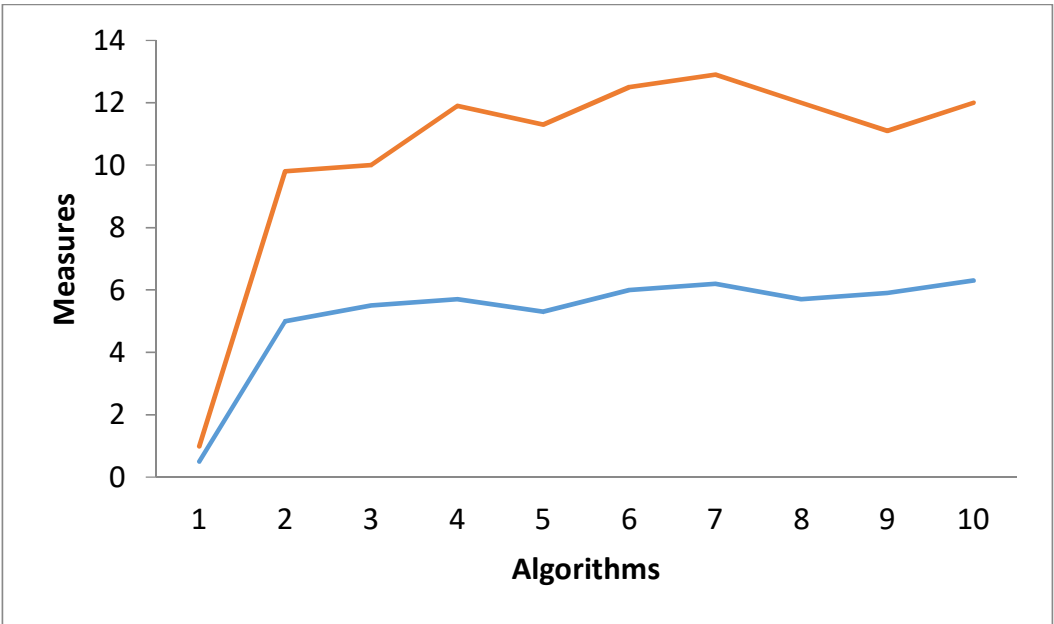

Figure 6: Comparison of Algorithms 


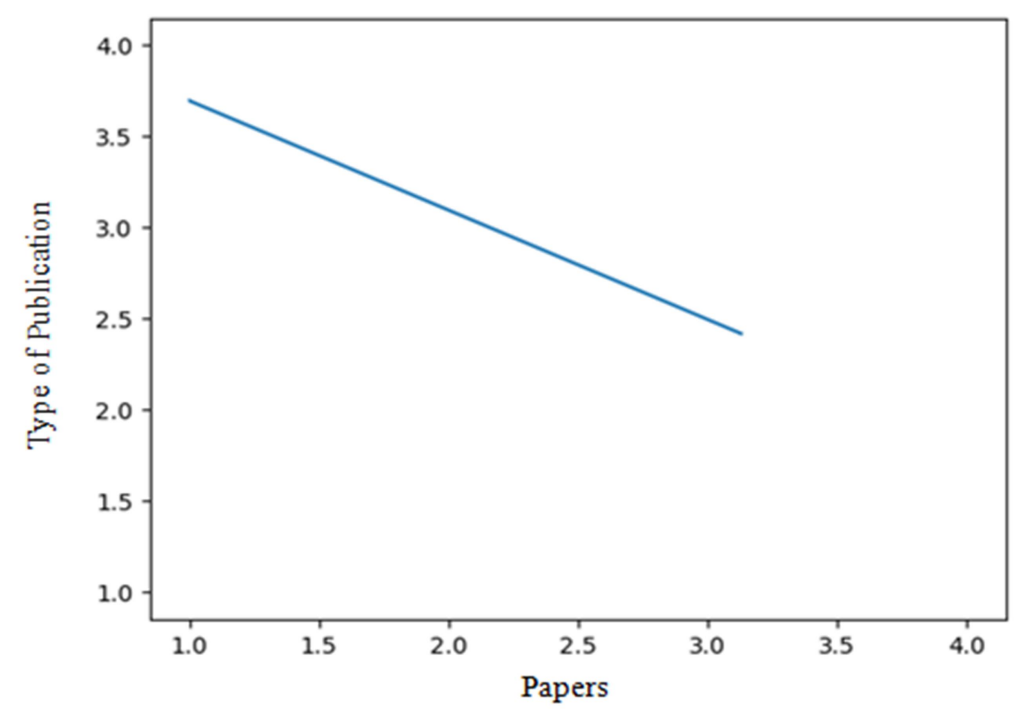

Figure 7: Papers Vs Types of Publication

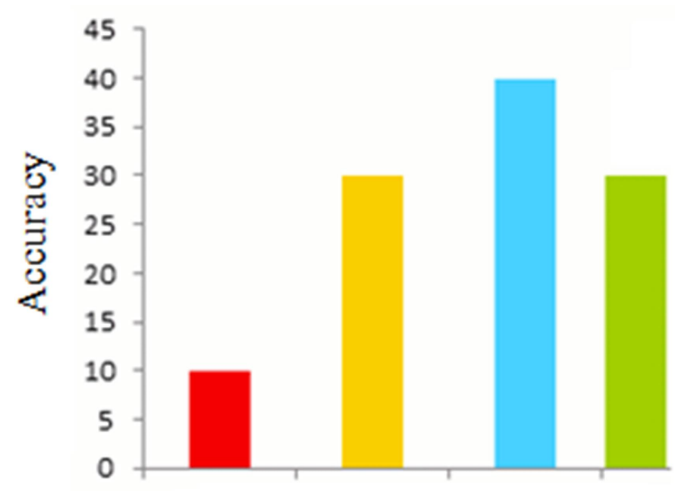

Algorithms

Figure 8: Random Based Algorithms Results

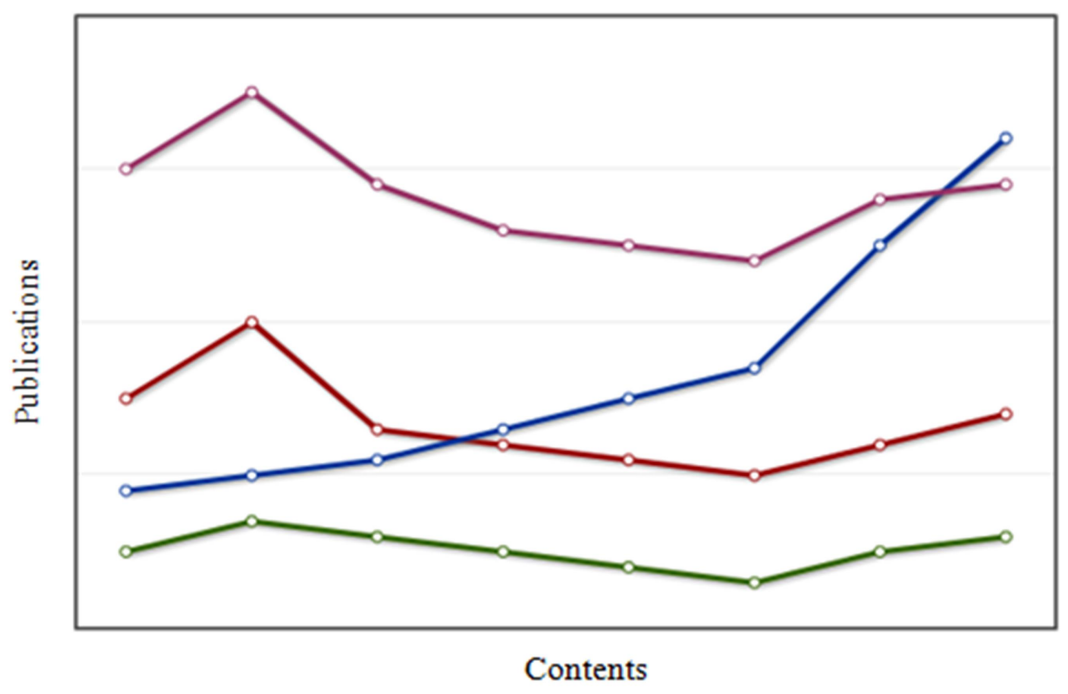

Figure 9: Results for Decision rule-based recognition 


\section{CONCLUSION}

A variety of connected phones, such as sensors, actuators, and other digital phones, were linked or placed for connectivity, engagement, or problem resolution. An amount of internet connected devices of Things are growing than ever. Interconnection of these gadgets has opened up new possibilities of intelligent systems, which is a rapidly increasing field of study. Confidentiality and anonymity were regarded to be among the most important challenges that academics to address among these prospects. Emerging civilizations have a clear need for amazing skills. Internet of Things (IoT) plays an important role as in amazing skills of these gadgets. Innovation has mostly concentrated on the economical well-being of humanity as well as environmental conservation. These devices communicate with one another, making human existence easier. The interconnection of these devices has opened up new possibilities of intelligent systems, which is a rapidly increasing field of study. Aggressors would be unable to disrupt that integrity of IoT network within smart city and safe data stream if proper safety changes are applied. A suggested research offers machine learning approach for safeguarding internet data centred on a gateway for connected phones or IoT networks, keeping in mind the security considerations of data usage for smart urban or IoT. For evaluation reasons, research implemented "Firewall" database. An approach's experimental results reveal that hybrid classification economically advantageous decision1 regulations or arbitrary forest, with hybrid version providing a recognition accuracy of 95.5 percent, 68.5 percent for prediction models, or 78.3 percent reliability for arbitrary forest. Several performance indicators such as error margin remember, $\mathrm{f}$ score, or accuracy are used to verify the validity of the given model. High precision level, and other performance measures, demonstrates overall impact of a proposed composite paradigm for protected data stream in connected devices.

\section{REFERENCES}

[1] Haghighi MS, Farivar F, Jolfaei A. A machine learning-based approach to build zero false-positive IPSs for industrial IoT and CPS with a case study on power grids security. IEEE Transactions on Industry Applications. $2020 \mathrm{Jul} 23$.

[2] Mandalari AM, Kolcun R, Haddadi H, Dubois DJ, Choffnes D. Towards automatic identification and blocking of non-critical iot traffic destinations. arXiv preprint arXiv:2003.07133. 2020 Mar 16. 
[3] Mandalari AM, Dubois DJ, Kolcun

R, Paracha MT, Haddadi H, Choffnes D. Blocking without Breaking: Identification and Mitigation of Non-Essential IoT Traffic. arXiv preprint arXiv:2105.05162. 2021 May 11.

[4] Ertam F. An efficient hybrid deep learning approach for internet security. Physica A: Statistical Mechanics and Its Applications. 2019 Dec 1; 535: 122492.

[5] Ahmad R, Alsmadi I. Machine learning approaches to IoT security: A systematic literature review. Internet of Things. 2021 Jan 29: 100365.

[6] Thakkar A, Lohiya R. A review on machine learning and deep learning perspectives of IDS for IoT: recent updates, security issues, and challenges. Archives of Computational Methods in Engineering. 2021 Jun; 28(4): 321143.

[7] Pavlović N, Šarac M, Adamović S, Saračević M, Ahmad K, Maček N, Sharma DK. An approach to adding simple interface as security gateway architecture for IoT device. Multimedia Tools and Applications. 2021 Aug 12: 1-6.
[8] Huang Y, Nazir S, Ma X, Kong S, Liu Y. Acquiring Data Traffic for Sustainable IoT and Smart Devices Using Machine Learning Algorithm. Security and Communication Networks. 2021 Jun 19; 2021.

[9] Ray AK, Bagwari A. IoT based Smart home: Security Aspects and security architecture. In2020 IEEE 9th International Conference on Communication Systems and Network Technologies (CSNT) 2020 Apr 10 (pp. 218-222). IEEE.

[10] Visoottiviseth V, Sakarin P, Thongwilai J, Choobanjong $\mathrm{T}$. Signature-based and Behaviorbased Attack Detection with Machine Learning for Home IoT Devices. In2020 IEEE Region 10 Conference (Tencon) 2020 Nov 16 (pp. 829-834). IEEE.

[11] Doshi R, Apthorpe N, Feamster N. Machine learning ddos detection for consumer internet of things devices. In2018 IEEE Security and Privacy Workshops (SPW) 2018 May 24 (pp. 29-35). IEEE.

[12] Anwar MN, Nazir M, Ansari AM. Modeling security threats for smart cities: A stride-based approach. Smart Cities- 
Opportunities and Challenges.

Springer. 2020 Apr 20: 387-96.

[13] Anwar RW, Abdullah T, Pastore

F. Firewall Best Practices for

Securing Smart Healthcare

Environment: A Review. Applied

Sciences. 2021 Jan; 11(19): 9183.

[14] Ghazanfar S, Hussain F, Rehman

AU, Fayyaz UU, Shahzad F, Shah

GA. Iot-flock: An open-source

framework for iot traffic

generation. In2020 International

Conference on Emerging Trends

in Smart Technologies (ICETST)

2020 Mar 26 (pp. 1-6). IEEE.

[15] Apthorpe N, Reisman D, Feamster

N. Closing the blinds: Four strategies for protecting smart home privacy from network observers. arXiv preprint arXiv:1705.06809.2017 May 18.

[16] Apthorpe N, Huang DY, Reisman D, Narayanan A, Feamster N. Keeping the smart home private with smart (er) iot traffic shaping. arXiv preprint arXiv:1812.00955. 2018 Dec 3.

[17] Susmita KS, Kailas DP. Portable Firewall for Data Security toward Secured Communication. gateways. 2021; 4: 5 . 